\title{
Análise Microestrutural de Arenito da Formação Rio do Rasto pela Microtomografia Computadorizada por raios $\mathrm{X}$
}

\section{Rio do Rasto Formation Sandstone's Microstructural Analysis by X Ray Micro Computed Tomography}

\author{
Alisson Henrique Dal Col${ }^{1}$, Paulo Henrique Gali ${ }^{2}$, Carlos Roberto Appoloni ${ }^{3}$
}

\begin{abstract}
Resumo
Neste trabalho são analisadas imagens microtomográficas do Arenito da Formação Rio do Rasto, obtidas pela metodologia de Microtomografia Computadorizada por raios X. As medidas foram realizadas com o microtomógrafo SkyScan, modelo 1172, e propriedades físicas de interesse foram obtidas, tais como: porosidade (fechada e conectada), distribuição de tamanho de poros, VER (Volume Elementar Representativo) e reconstrução 3D. Esta é uma técnica de análise não destrutiva e, por fornecer informações internas das rochas sem destruí-las, é foco de atenção de empresas petrolíferas. Para a aquisição de imagens foi utilizada uma resolução espacial de 5,87 $\boldsymbol{\mu m}$ com uma tensão de $70 \mathrm{kV}$ no tubo de raios X. As projeções foram reconstruídas - gerando as seções 2D - onde, então, foram minimizados alguns dos artefatos nas imagens tomográficas. Com os softwares Imago e CTAn foram analisados os parâmetros físicos sobre uma região de interesse (ROI). Aplicado o limiar médio de 67(2) em uma escala de cores de 8 bits, se obteve - pelo software Imago - uma porosidade absoluta de 13,54(66) \%, onde $12,58(74) \%$ são poros conectados. Baseando-se na porcentagem de poros conectados e na distribuição de tamanho de poros, infere-se que esta amostra poderia conter uma razoável permeabilidade. O VER foi alcançado com uma aresta de 2,900 $\mathrm{mm}$. Comparando com trabalhos anteriores, notou-se que esta amostra possui um algo grau de variabilidade.
\end{abstract}

Palavras-chave: Formação Rio do Rasto. Porosidade. Distribuição de Tamanho de Poros. Rochas Reservatório. Microtomografia. Raios X.

\begin{abstract}
In this paper, microtomographic images of Rio do Rasto formation's sandstone are analyzed, obtained by X Ray Micro Computed Tomography methodology. They were were performed with SkyScan 1172 microCI scanner and, some physics properties were obtained, such as: porosity (closed and connected), pore size distribution, REV (Representative Elementary Volume), and 3D reconstruction. This is a nondestructive method and it provides internal information about rocks without destroying them, it's strongly used by petroleum industries. About image acquisition, the spatial resolution was $5.87 \boldsymbol{\mu m}$ and the X-ray tube voltage was adjusted to $70 \mathrm{kV}$. Then, the projections were reconstructed - generating 2D sections - and artifacts were reduced at tomographic images. With Imago and CTAn softwares, physics parameters were analyzed on the Region Of Interest (ROI). When the standard threshold (67) was applied on the 8 bits color scale, we obtained - through Imago software - 13,54(66) \% of total porosity, where $12,58(74) \%$ is related to connected pores. Considering the percentage of connected pores and the pore size distribution, it infers that this sample might show a reasonable permeability. VER was reached with a $2,900 \mathrm{~mm}$ cube's edge. Comparing with previous works, it noticed that this rock has a great level of variability.
\end{abstract}

Keywords: Rio do Rasto's Formation. Porosity. Pore Size Distribution. Reservoir Rocks. Microtomography. X-ray.

\footnotetext{
${ }^{1}$ Mestrando Junto ao Departamento de Física, Universidade Estadual de Londrina; alissonhdc@hotmail.com

${ }^{2}$ Professor da Escola Estadual Virgílio Capoani, Lençóis Paulista, São Paulo; paulo_gali@hotmail.com

${ }_{3}^{3}$ Docente do Departamento Física da Universidade Estadual de Londrina; appoloni@uel.br
} 


\section{Introdução}

A caracterização de rochas-reservatório quanto à sua microestrutura é uma das mais importantes etapas concernentes à análise de sistemas petrolíferos. Para este fim, várias técnicas são empregadas como, porosimetria por Mercúrio (Hg), análise de imagens bidimensionais por microscopia de lâminas delgadas ou por imagens tridimensionais via Tomografia Computadorizada (CT) e/ou suas variantes: Microtomografia Computadorizada ( $\mu$ CT) e Nanotomografia Computaorizada (n $\mathrm{CT}$ ). Quando se necessita fazer uma análise visual dos parâmetros microestruturais dessas rochas, a lâmina delgada restringe-se apenas a visualizações bidimensionais. Por outro lado, a $\mu \mathrm{CT}$ por raios $\mathrm{X}$ destaca-se por proporcionar uma visualização tridimensional desses parâmetros e, ainda mais, possibilita resultados quantitativos dos parâmetros (FERNANDES et al., 2012).

Um dos mais importantes parâmetros físicos que se pode obter de uma rocha é a porosidade $(\phi)$. Para Nimmo (2004), a porosidade é a fração volumétrica total da rocha que é tomada por espaços porosos (eq. 1). Por sua vez, os espaços porosos são, geralmente, determinados em termos dos poros individuais - um conceito artificial que permite a quantificação deste parâmetro essencial. Poros, portanto, são espaços preenchidos ou não por fluídos (gás, líquido) no interior da rocha, os quais podem permear toda ela e estar conectados uns com os outros ou não (Porosidade aberta/conectada e porosidade fechada, respectivamente). Temos, então,

$$
\phi=\mathrm{V}_{\mathrm{p}} \mathrm{V}_{\mathrm{V}}
$$

onde $\mathbf{V}_{\mathbf{p}}$ refere-se ao volume total de poros e $\mathbf{V}$ ao volume total da rocha.

Outro importante parâmetro físico é a Distribuição de Tamanho de Poros (DTP). Para Nimmo (2004), a DTP é a abundância relativa de cada tamanho de poro em um volume representativo da rocha. Ou seja, ela é definida como a distribuição estatística dos raios das maiores esferas que podem ser construídas dentro de um poro em um dado ponto. Este tratamento é feito via algoritmos baseados em Morfologia Matemática (GONZALEZ, 2002).

Por último, a permeabilidade de uma rocha é a medida da resistência ao fluxo de um fluído através dela. Se for necessária uma pressão muito alta para que um fluído permeie através da rocha, então ela possuirá baixa permeabilidade. Por outro lado, se o fluído caminha através da amostra facilmente, então esta possuirá alta permeabilidade (GEOMORE, 2015). Assim, se uma rocha possuísse uma rede porosa altamente conectada, e o raio dessas conexões fosse grande o bastante (quando comparado ao raio dos poros), então, provavelmente, o fluído movimentar-se-ia no interior da rocha com facilidade, ou seja, esta amostra geológica possuiria alta permeabilidade.

Assim, como a microscopia de lâminas delgadas, a $\mu$ CT também fornece imagens 2D das secções transversais das amostras, porém, de uma forma bem mais completa. Mais ainda, o grande potencial da microtomografia é a reconstrução em imagens 3D. Entretanto, nem sempre é possível criar essas imagens no tamanho desejado, pois este processo requer um grande potencial computacional. Então, tornase necessário determinar o Volume Elementar Representativo (VER) da amostra. Para o caso de rochas com uma rede de poros homogênea, o VER é determinado como o menor volume onde a porosidade torna-se independente da escala, e representa, precisamente, a amostra em sua totalidade (BEAR, 2002).

A importância da determinação do VER está em servir como parâmetro para quem for estudar rochas assemelhadas e, por outro lado, a questão das simulações, i.e., a simulação de percolação dentro de rochas. Desta forma, quanto menor for 
o volume que represente a amostra, menos tempo demandará o processo de simulação.

A rocha analisada neste trabalho se caracteriza como rocha reservatório do tipo arenito. Arenitos são rochas arenosas e uma das mais encontradas na superfície terrestre. Elas são constituídas substancialmente de partículas ou grânulos de quartzo detrítico, sub-angulares ou angulares. O cimento pode ser sílica, carbonato de cálcio, substâncias ferruginosas, etc (FERNANDES, 2009). O valor médio da porosidade para arenitos, de acordo com Barrel (1914), oscila em torno de $14,8 \%$.

Portanto, o objetivo deste trabalho baseia-se em caracterizar os parâmetros microestruturais do arenito da formação Rio do Rasto, tais como a porosidade, distribuição de tamanho de poros, e o VER, através da metodologia de microtomografia por raios $\mathrm{X}$,uma vez que esta é uma metodologia não destrutiva e eficiente para este tipo de análise. Por outro lado, objetiva-se compreender o grau de variabilidade que este tipo de rocha apresenta, comparando com trabalhos anteriores.

\section{Materiais e Métodos}

As medidas foram realizadas em um microtomógrafo fabricado pela empresa Bruker, modelo SkyScan 1172. Este equipamento, atualmente, está instalado no Laboratório de Análise por Raios X (LARX) da Universidade Estadual de Londrina (UEL), Londrina - PR.

A amostra é oriunda da formação Rio do Rasto pertencente à Bacia do Paraná, que engloba os estados de Santa Catarina, Paraná e Rio Grande do Sul. Esta formação, de idade neo-permianatriássica, apresenta espessura de até $400 \mathrm{~m}$ nos afloramentos da borda leste da Bacia Sedimentar do Paraná. A formação constitui-se de arenitos, siltitos e, secundariamente, argilitos. Os arenitos formam lentes relativamente curtas, em geral, com menos de $3 \mathrm{~m}$ de espessura, podendo chegar a 10m. Nos afloramentos, há predomínio das cores vermelhas, podendo ocorrer tonalidades de chocolate, púrpura e verde (BASTOS, 2008).

O microtomógrafo é constituído por um tubo de raios $\mathrm{X}$, mesa para o deslocamento da amostra, detector e alguns softwares de aquisição e reconstrução das imagens tomográficas. A geometria desse equipamento é com fonte-detector fixos, enquanto a amostra se movimenta com dois graus de liberdade de translação (vertical e horizontal) e um de rotação em torno do eixo de simetria ortogonal à base do porta amostra. A translação vertical é utilizada para controlar qual região relacionada à altura da amostra estará no campo de visão (FOV) do detector CCD, a horizontal relaciona-se com a resolução espacial da imagem tomográfica, e a rotação apresenta-se durante o processo de aquisição das projeções.

O aparelho é equipado com um tubo de raios $\mathrm{X}$ de anodo de Tungstênio (W) que trabalha com um intervalo de tensão de $20 \mathrm{kV}$ a $100 \mathrm{kV}$ e de corrente elétrica de $0 \mu \mathrm{A}$ a $250 \mu \mathrm{A}$. O sistema de detecção é constituído por uma câmera CCD de 11 megapixels, 12 bits, refrigerada por efeito Peltier e acoplada a um dispositivo cintilador, atingindo uma resolução máxima de $0,9 \mu \mathrm{m}$.

As projeções foram adquiridas pelo SkyScan 1172 conforme descrito por Appoloni et al. (2010). Desse modo, as configurações utilizadas foram de $70 \mathrm{kV}, 141 \boldsymbol{\mu A}$, em passos angulares de $0,4^{\circ}$ em uma tomada de dados em $360^{\circ}$ e atenuação média do feixe de $31,8 \%$. Utilizouse a média de 3 medidas para cada passo angular com uma resolução espacial de 5,87 $\boldsymbol{\mu m}$ em um tempo total de 3,6 h.

Posteriormente, as projeções foram reconstruídas em secções 2D pelo software NRecon. Porém, anteriormente, utilizou-se o mesmo software para eliminar e/ou minimizar alguns artefatos comumente encontrados. 
Figura 1: Perfil de atenuação de uma projeção tomada com tensão de $70 \mathrm{kV}$ e $141 \mu \mathrm{A}$ de corrente elétrica.

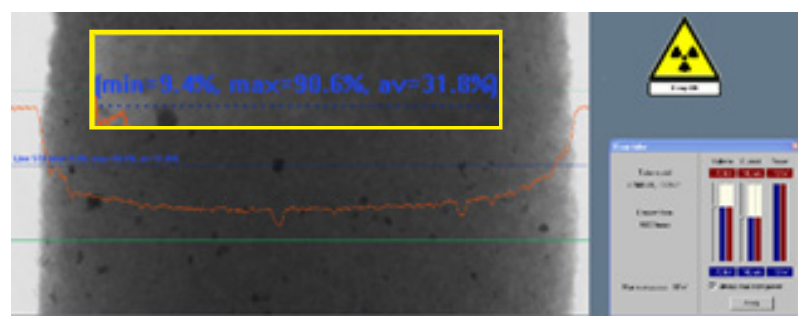

Fonte: dos autores.

Em seguida, através do software CTAn, foram recortadas das secções 2D a maior região de interesse possível em forma de um quadrado (ROI), cuja dimensão das arestas foi de 5,526 mm (Figura 2). Após o recorte, importaram-se as imagens para o software Imago onde foram convertidas em tons de cinza de 8-bits (Figura 3-a).

Figura 2: Imagem tomográfica $n^{\circ} 194$ e respectiva região de interesse na moldura em amarelo.

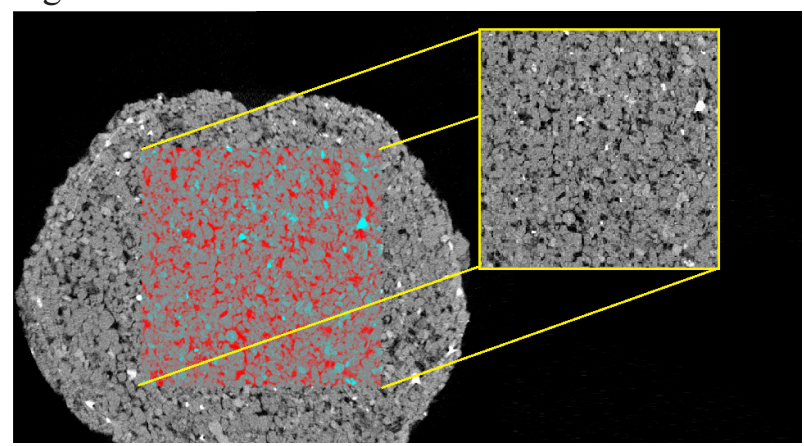

Fonte: dos autores.

De posse dos ROI's em 8 bits, o estudo de um limiar de tom de cinza adequado foi realizado. De acordo com o estudo realizado por Moreira (2011), um dos métodos que melhor se enquadrou nas características da amostra estudada neste artigo foi o baseado no método de Otsu, cujo comando no MATLAB é o graythresh. Entretanto, a escolha automática de um threshold costuma não ser muito eficiente, assim, a intervenção por uma escolha manual foi utilizada.

Para a escolha manual, o olho humano é limitado a distinguir, aproximadamente, 30 tonalidades. Para minimizar esta limitação optou-se por acrescentar um desvio de duas unidades para mais e duas para menos do valor de limiar de tom de cinza escolhido pelo operador do software. Assim, ter-se-á também desvios na porosidade total 3D (bem como na porosidade aberta e fechada) os quais serão calculados através da Equação 2.

$$
\mathrm{D}=\frac{\phi\left(\mathrm{T}_{\text {máx }}\right)-\phi\left(\mathrm{T}_{\min }\right)}{2}
$$

onde $\phi\left(T_{\max }\right)$ e $\phi\left(T_{\min }\right)$ são as estimativas da porosidade nos limiares de $T_{\operatorname{máx}}$ e $\mathrm{T}_{\min }$, respectivamente, sendo que $\mathrm{T}_{\min }$ é o limiar duas unidades menor e $\mathrm{T}_{\operatorname{máx}}$ duas unidades maior que o limiar escolhido.

Seguidamente, binarizou-se as imagens em fase sólida (pixel preto) e fase poro (pixel branco) (Figura 3-b). Por fim, calculou-se a porosidade 3D total da amostra bem como a distribuição de tamanho de poros via método de abertura e fechamento (combinação dos processos de erosão e dilatação em imagens digitais) com elementos estruturantes empregando o software Imago (FERNANDES, 2009 p. 67).

Figura 3: Secção 2D n 136: (a) Imagem em 8 Bits (tons de cinza); (b) Imagem binarizada com um limiar de 67 onde regiões brancas representam poros.

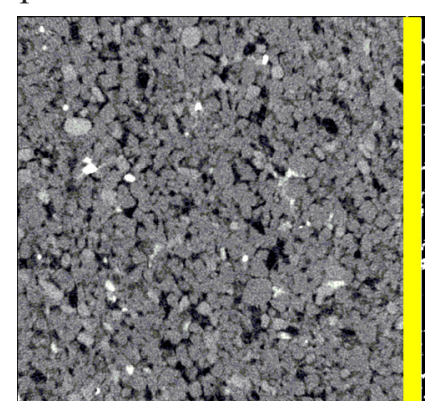

(a)

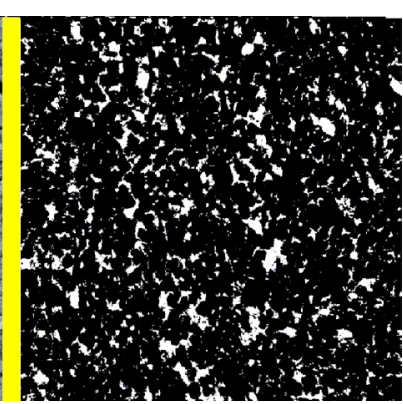

(b)
Fonte: dos autores.

Para a determinação do VER referente à porosidade, o método consistiu em utilizar crescentes subvolumes cúbicos (Figura 4) com arestas medindo $290 \mu \mathrm{m}, 580$ $\mu \mathrm{m}, 870 \mu \mathrm{m}, 1160 \mu \mathrm{m}, 1450 \mu \mathrm{m}, 1740 \mu \mathrm{m}, 2030 \mu \mathrm{m}$, 
$2320 \mu \mathrm{m}, 2610 \mu \mathrm{m}, 2900 \mu \mathrm{m}, 3480 \mu \mathrm{m}$ e $4060 \mu \mathrm{m}$. Este procedimento se repetiu para cinco regiões diferentes da amostra: superior esquerda (SE), superior direita (SD), inferior esquerda (IE), inferior direita (ID) e central (CE). Para a escolha do VER, a porosidade do menor subvolume deveria apresentar um desvio relativo menor ou igual a $1 \%$ com relação à porosidade total $3 \mathrm{D}$.

Figura 4: Localização dos subvolumes criados na fatia $\mathrm{n}^{\circ} 123$ para a determinação do VER

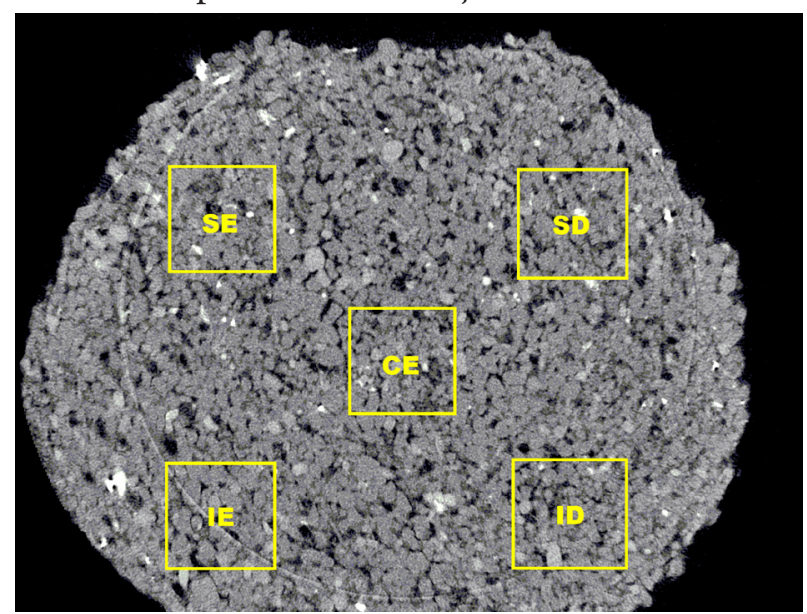

Fonte: dos autores.

\section{Resultados e Discussões}

O processo automático de obtenção do limiar apresentou um valor de 95, o que superestimava as regiões porosas. Dessa maneira, optou-se pela escolha manual e o valor de limiar escolhido para os ROI's foi de 67 (2) (Figura 5).

Figura 5: Secção 2D n ${ }^{\circ} 152$ - Exemplificação do processo de binarização no Imago com a inversão, branco (fase poro) e preto (fase sólida).

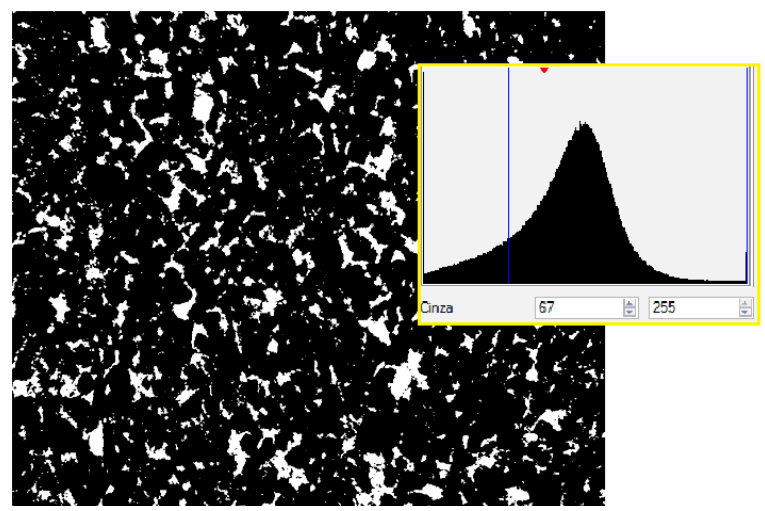

Fonte: dos autores.
Através deste valor de limiar, obteve-se que a porosidade total 3D do arenito foi de 13,54(66) $\%$. Porém, contido nesse valor, constatou-se que $12,58(74) \%$ são poros conectados e 1,09(9) \% são poros fechados. Por outro lado, constata-se pela Figura 6 que grande parte da porosidade é determinada por poros cujos raios estão entre $10 \mu \mathrm{m}$ e $30 \mu \mathrm{m}$. Estes representam, aproximadamente, $66 \%$ da quantidade total de poros presentes no volume analisado.

Figura 6: Distribuição dos raios dos poros para o volume analisado com arestas de $5,526 \mathrm{~mm}$.

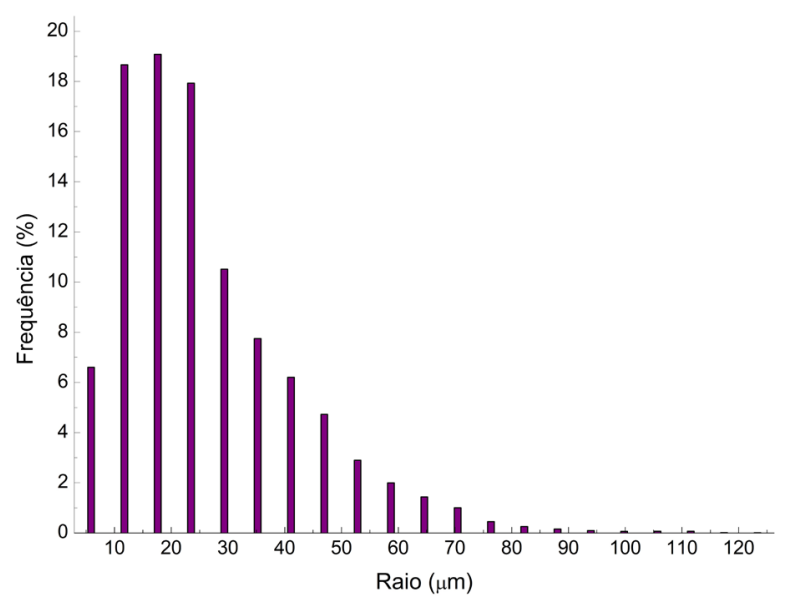

Fonte: dos autores.

Vale ressaltar que esta medida foi realizada com uma resolução espacial de $5,87^{\mu \mathrm{m}}$, ou seja, a câmera $\mathrm{CCD}$, distingue somente objetos maiores ou iguais a essa resolução. Desta forma, pode haver uma pequena fração de poros com raios menores que $5,87^{\mu \mathrm{m}}$, mas, para detectá-los seria necessária uma medida com resolução espacial menor.

De posse dos subvolumes adquiridos pelo processo representado na Figura 4 e tomando como comparação a porosidade total $3 \mathrm{D}$ da amostra para o maior ROI cuja aresta foi de 5,526 $\mathrm{mm}$, pode-se verificar na Figura 7 quais regiões e quais subvolumes representam a amostra quanto à porosidade dela. 
Figura 7: Estabilidade da porosidade dos subvolumes com a variação de suas respectivas arestas em diferentes regiões da amostra.

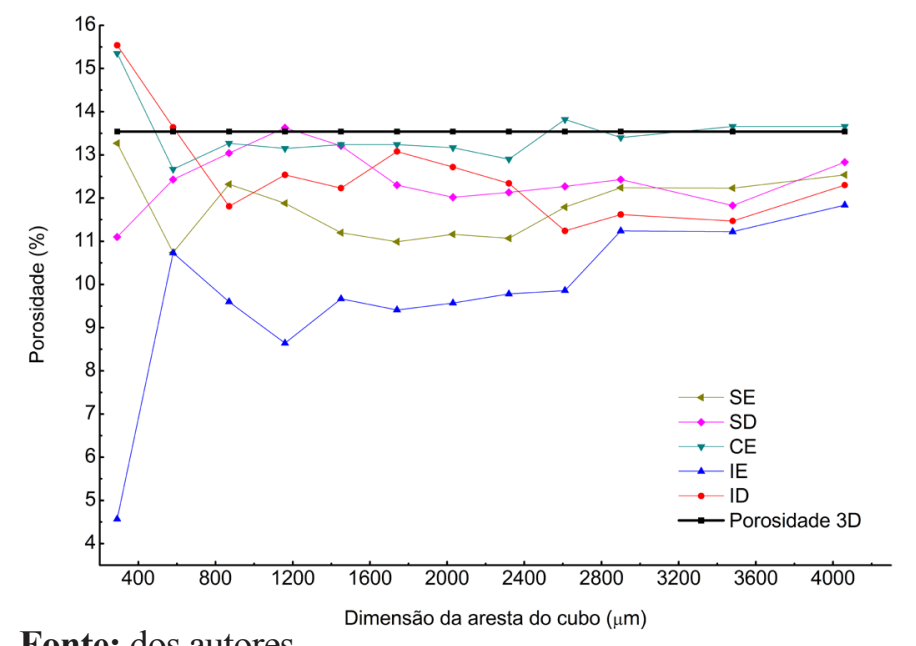

Fonte: dos autores.

Observa-se que, para cubos de arestas medindo menos que 2800 , flutuações aleatórias na porosidade são observadas para algumas regiões, i.e., IE, ID, SD, SE. Estas flutuações estão associadas com o grau de heterogeneidade da porosidade contida naqueles subvolumes, uma vez que se constatou, através da Figura 6, que há uma considerável quantidade de poros com raios grandes, tomando como base a dimensão das arestas dos cubos.
Assim, um poro pode estar totalmente dentro como, também, fora do subvolume, acarretando em uma variação brusca da porosidade à medida que se cresce o volume do cubo. Pode-se visualizar essa variação ao analisar a Figura 8, a qual apresenta o erro relativo à porosidade total 3D de 13,54(66)\%. Exceto a região $\mathrm{CE}$, todas as outras situam-se fora da condição de obter um erro relativo menor ou igual a $1 \%$. Porém, a região central (CE) obteve, estatisticamente, uma estabilidade aceitável a partir do subvolume cuja aresta mede 2,900 $\mathrm{mm}$.

Figura 8: Erro relativo da porosidade dos cubos (subvolumes) com relação à porosidade total $3 \mathrm{D}$ do maior VOI (Volume de Interesse).

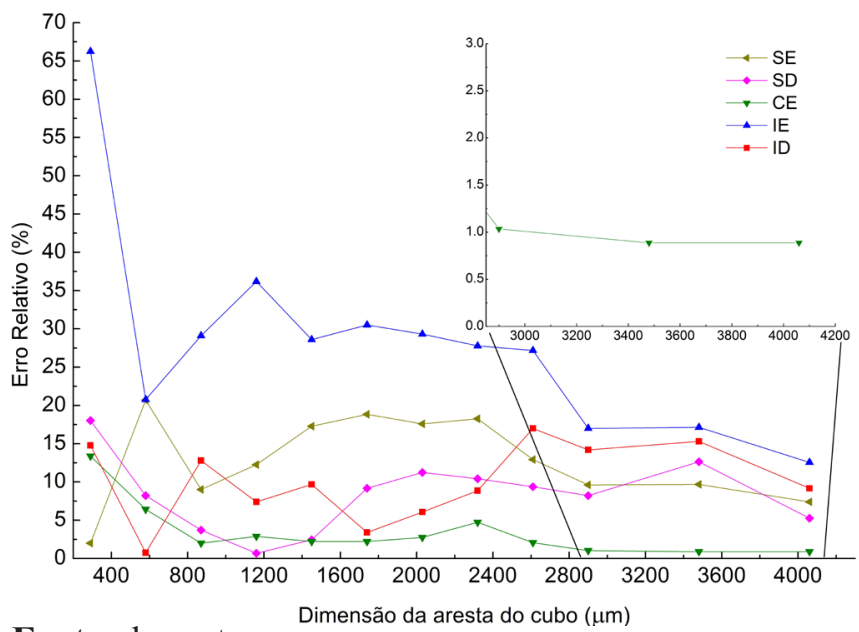

Fonte: dos autores. 
Portanto, atingiu-se o VER e o menor subvolume que representa a amostra quanto à porosidade possui uma aresta de $2,900 \mathrm{~mm}$. Para este VOI, a porosidade 3D total estimada foi de $13,40(66) \%$, onde $12,55(71) \%$ corresponde a poros conectados e 0,97(6) \% refere-se a poros fechados. Assim, estatisticamente, as porosidades conectada e fechada também se correspondem.

De posse das dimensões do VER, foi possível gerar o volume 3D para uma análise qualitativa do interior da amostra (Figura 9).

Figura 9: Representação em 3D do VER. (a) fase poro; (b) fase sólida.

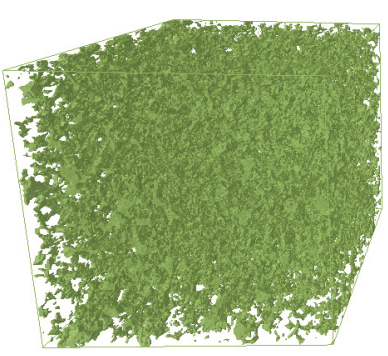

(a)

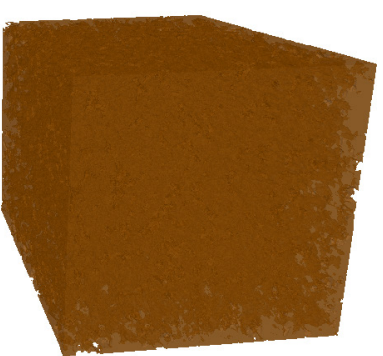

(b)
Fonte: dos autores.

$1,45 \mathrm{~mm}$

Tanto pelo VER quanto pelo VOI de $5,526 \mathrm{~mm}$ de aresta, viu-se que, aproximadamente, $94 \%$ da porosidade total $3 \mathrm{D}$ corresponde à porosidade conectada. Desta forma, pode-se inferir que esta amostra pode apresentar uma propensão à movimentação de fluídos dentro dela. Claro, há necessidade de conhecer qual o diâmetro das gargantas que conectam os poros. Entretanto, como a maioria dos poros são conectados, se pode estimar o raio das gargantas analisando a distribuição de tamanho de poros apresentado na Figura 6. Através dela, constata-se que a maioria dos poros possui raio suficientemente grande. Assim, infere-se que esta amostra poderia apresentar uma razoável permeabilidade.
De acordo com Fernandes (2009), um arenito da mesma formação foi analisado. Neste, a amostra foi microtomografada em uma resolução espacial de 2,9 $\mu \mathrm{m}$. A porosidade adquirida foi de 4,2(1) \% e, na distribuição de tamanho de poros, $92 \%$ dos poros encontra-se com raios entre 2,9 e 20,6 $\mu \mathrm{m}$, com uma moda em 11,7 $\mu \mathrm{m}$. O VER encontrado para esta amostra foi aquele com aresta igual a $1176 \mu \mathrm{m}$.

A diferença da porosidade observada entre os dois trabalhos pode ser devida a dois fatores. $\mathrm{O}$ primeiro e, provavelmente, o mais importante, o fato de serem duas amostras diferentes e de coleta independente daquela formação rochosa. Considerando a heterogeneidade dessas amostras geológicas, estamos observando a variabilidade das mesmas. O segundo fator, e de segunda ordem, pode estar na resolução espacial utilizada em cada uma dessas análises, bem como, no valor de limiar de tom de cinza empregado.

Dessa forma, como o arenito estudado por Fernandes (2009) foi analisado em uma resolução espacial menor, esperar-se-ia obter uma porosidade total 3D maior que $13,54(66) \%$, uma vez que, nesta resolução, poros menores seriam identificados, contribuindo, portanto, para a porcentagem de espaços vazios dentro da amostra. Entretanto, não foi esse o observado e pode-se atribuir esta diferença ao grau de variabilidade dessas rochas, são amostras geológicas altamente heterogêneas, o que fica ainda mais claro ao observar a Figura 10.

Nesta figura, observa-se que o arenito estudado por Fernandes (2009) é muito mais fechado do que o analisado por nós, ou seja, a porosidade do arenito de Fernandes deveria apresentar uma porosidade menor, o que é fato. Além disso, nota-se que a composição de ambas as rochas se diferem muito, pois apresentam padrões diferentes de tonalidade de cinza, o que, embora possuam a mesma formação geológica, comprova, ainda mais, a diferença entre as duas amostras comparadas. Assim, também, não há motivos para que o VER de ambas sejam iguais. 
Figura 10: Comparação entre as imagens microtomográficas das rochas dos dois trabalhos: (a) Fatia 108 (presente trabalho); (b) Fatia 110 (adaptado de FERNANDES, 2009).

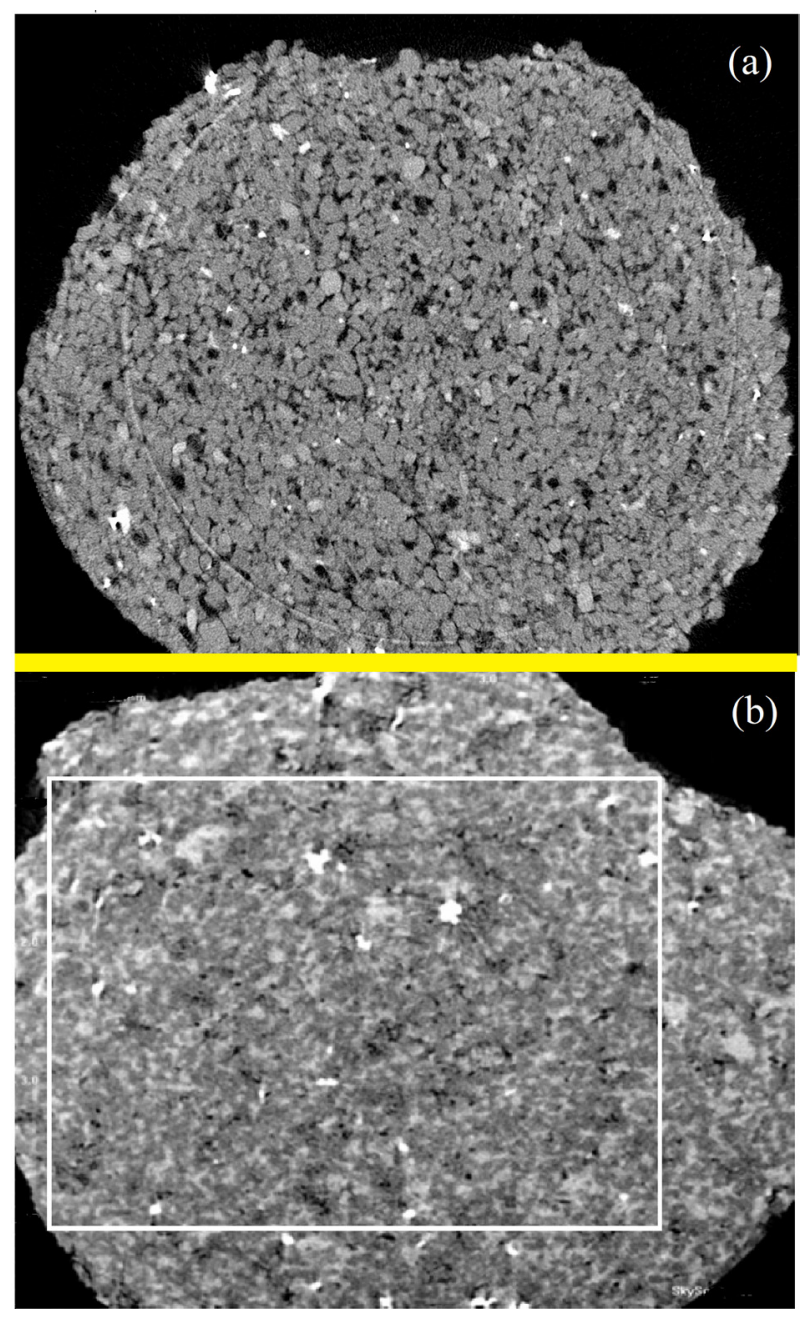

\section{Conclusões}

Através da microtomografia por raios $\mathrm{X}$, analisou-se a rocha reservatório do tipo Arenito pertencente à formação Rio do Rasto e constatouse que esta rocha, para uma resolução espacial de $5,87 \mu \mathrm{m}$, possui uma porosidade razoável de $13,54(66) \%$. Dentro desse valor, 12,55(71) $\%$ corresponde a poros conectados e 0,97(6) \% refere-se a poros fechados, ou seja, quase 94 $\%$ da porosidade total 3D é composta por poros conectados entre sí. Como a porosidade conectada representa a maior parte da porosidade total $3 \mathrm{D}$ e, de acordo com a distribuição de tamanho de poros, a maioria dos poros possui raio entre 10 e $30 \mu \mathrm{m}$ . Então, pode-se inferir que esta amostra poderia apresentar uma razoável permeabilidade.

Observou-se também que o Volume Elementar Representativo do Arenito quanto a porosidade situou-se no cubo cuja aresta mede $2,900 \mathrm{~mm}$. Baseado no VER, a porosidade total 3D foi estimada em 13,40(66) $\%$, onde $12,55(71) \%$ corresponde a poros conectados e $0,97(6) \%$ refere-se a poros fechados. Assim, estatisticamente, as porosidades conectada e fechada são iguais às do maior VOI. Portanto, um processo de simulação de percolação poderia ser realizado sobre este VER da rocha, demandando menos tempo de processamento, quando comparado ao maior VOI da amostra.

Comparado ao trabalho executado por Fernandes (2009), uma rocha da mesma formação foi analisada e resultados totalmente diferentes foram obtidos. A porosidade, por exemplo, foi menor para o trabalho de Fernandes do que para o nosso, mesmo ele realizando a aquisição das projeções em uma resolução espacial menor (o que deveria apresentar a maior porosidade). Ao analisar a Figura 10, notase com clareza que o arenito de Fernandes é bem mais compacto do que o do presente trabalho, o que justifica a porosidade de 4,2(1) \%. Através desses valores, se pode notar o grau de variabilidade que essas rochas possuem, embora sejam oriundas da mesma formação. Dessa forma, não tem por que se esperar um VER semelhante entre as duas análises.

Por conseguinte, baseando-se nos dados encontrados para o Arenito analisado neste trabalho e o analisado por Fernandes (2009), se pode inferir que ele possuiria uma grande propensão para extração eficiente de fluídos, é um tipo de rocha que, sob a mesma formação do Rio do Rasto, pode apresentar um alto grau de variabilidade em diferentes pontos de extração da amostragem. Com a obtenção do VER, tornar-seia mais viável a simulação de intrusão/extração de 
fluídos nesta rocha, uma vez que o volume a ser simulado (VER) seria menor do que o maior VOI possível para esta amostra. Assim, menor seria o tempo exigido para executar esta simulação, visto que este tipo de tarefa exige grande capacidade computacional e tempo de processamento.

Além disso, a micro-CT mostrou-se uma ferramenta altamente competente na caracterização dos parâmetros físicos destas amostras, sem contar no fato de ser uma metodologia não destrutiva. Metodologias alternativas que buscam uma caracterização dos mesmos parâmetros físicos, seriam altamente destrutivas às amostras, e.g., microscopia de lâminas delgadas.

\section{Referências}

APPOLONI, C. R.; MOREIRA, A. C.; MARQUES, L. C.; FERNANDES, J. S.; NAGATA, R.; Roteiro para Utilização do Microtomógrafo Skyscan 1172: Aquisição de Dados, Reconstrução e Análise de Imagens. Publicação Técnica do Laboratório de Física Nuclear Aplicada-UEL, v. 14, n. 02, 2010, $22 \mathrm{p}$.

BARREL, J. The strength of the earth's crust. Jour Geology, v. 22, p. 214. 1914.

BASTOS, R. O.; Radioatividade de rochas provenientes das formações geológicas pertencentes à Bacia Hidrográfica do rio Tibagi. Tese (Doutorado). Depto. De Física. Universidade Estadual de Londrina, Londrina, 2008.

BEAR, J.; Dynamics of Fluids in Porous Media, Dover, New York. 2002.

BREWER, R.; Fabric and mineral analysis of soils. New York, NY: Krieger, 1964. 482 p. Division of Soils, CSIRO, Australia.

FERNANDES, C. P.; MOREIRA, A. C.; MANTOVANI, I. F.; APPOLONI, C. R.; FERNANDES, J. S.; SOUZA, M. K.; SANTOS, V. S. S. Caracterização do sistema poroso de rochasreservatório com microtomografia computadorizada de raios X. B. Geoci. Petrobras. Rio de Janeiro, v. 20, n. 1/2, p. 129-144, nov. 2012.
FERNANDES, J. S. Caracterização Microestrutural do Espaço Poroso de Rochas Reservatório ds Bacia do Rio Tibagi por Microtomografia de raios $X$. Tese (Doutorado). Depto. De Física. Universidade Estadual de Londrina, Londrina, 2009.

GEOMORE. Porosity and Permeability. 2015. Disponível em: <http://www.geomore.com/ porosity-and-permeability-2/>. Acesso em 28 out. 15

GONZALEZ, R. C.; WOODS, R. E.; Digital Image Processing. New Jersey: Prentice-Hall, 2002. 799 p.

MOREIRA, A. C.; Métodos para segmentação binária em imagens em tons de cinza. LFNATEC - Publicação Técnica do Laboratório de Física Nuclear Aplicada. v. 15, n² 2. Out 2011.

NIMMO, J. R. Porosity and Pore Size Distribution. Encyclopedia of Soils in the Environment. London, Elsevier, v. 3, p. 295-303. 2004.
Recebido em 11Março, 2015 - Received on March 11, 2015 Aceito em 07 Abril, 2016 - Accepted on April 07, 2016 
